\title{
NOUVELlE
}

\section{Les protéines de la famille $\mathrm{Bcl}-2$ à $\mathrm{BH} 3$ seulement}

Muriel Priault, Stephen Manon

> L'existence et la pérennité des organismes pluricellulaires reposent principalement sur la capacité des cellules de communiquer entre elles. Dès le stade embryonnaire, cette communication s'avère d'une absolue nécessité pour le maintien de l'homéostasie. Tout au long de sa vie, une cellule perçoit des signaux extérieurs et intérieurs qui lui imposent son rythme de croissance, lui indiquent comment se différencier et comment coordonner ses diverses activités avec celles des cellules voisines, et finalement comment mourir sans constituer un danger potentiel pour l'organisme. L'apoptose désigne cette mort cellulaire programmée. Elle est la voie physiologique d'élimination cellulaire, qui survient en l'absence de toute maladie et qui fait partie intégrante du programme de développement des organismes.

L'observation de l'allongement de la durée de vie des cellules humaines dans lesquelles la protéine $\mathrm{Bcl}-2$ est surexprimée a rapidement permis de suspecter un lien avec un programme apoptotique défectueux. Par la suite, des alignements de séquences de résidus d'acides aminés ont été systématiquement pratiqués sur les protéines interagissant fonctionnellement et/ou physiquement avec $\mathrm{Bcl}-2$ et la terminologie de «domaines d'homologie avec $\mathrm{Bcl}-2$ » (ou domaines BH) a été adoptée pour désigner les zones conservées entre les membres de cette famille de protéines régulatrices de l'apoptose, dont certaines sont pro-apoptotiques et d'autres sont anti-apoptotiques [1]. L'absence de toute autre signature structurale permettant de prédire les mécanismes moléculaires du fonction- nement de ces protéines a motivé l'étude du rôle de chacun des quatre domaines BH identifiés à ce jour.

Une sous-famille de la famille $\mathrm{Bcl}-2$ est représentée par les protéines dites « à $B H 3$ seulement » et compte actuellement une dizaine de membres (Bad, Bbc3, Bid, Bik, Bim, Blk, Bmf, Hrk, Noxa, p193 et Puma). Ces protéines ne possèdent qu'un seul des quatre domaines d'homologie et sont toutes pro-apoptotiques. Leur capacité d'interagir in vitro avec les membres antiapoptotiques de la famille a longtemps laissé supposer qu'elles n'avaient qu'un rôle potentialisateur de l'apoptose, consistant en l'inhibition des protéines anti-apoptotiques par l'établissement d'interactions physiques avec elles. Cependant, des études récentes ont permis de mieux appréhender les particularités de fonctionnement de chacune et même, pour certaines, de leur attribuer des fonctions propres. L'activation de ces protéines est dépendante de régulations transcriptionnelles et posttraductionnelles. L'élucidation des mécanismes mis en jeu par les cellules pour régler l'activité de ces protéines révèle peu à peu la complexité mise au service de l'efficacité du contrôle du programme apoptotique.

Une stratégie classiquement mise en jeu par les cellules saines pour maintenir inactives les « protéines à $\mathrm{BH} 3$ seulement » est de les détourner de leur capacité de former des hétérodimères avec les protéines anti-apoptotiques de la famille $\mathrm{Bcl}-2$. En provoquant leur interaction avec d'autres composants cellulaires, la cellule assure donc leur séquestration jusqu'à la perception d'un
Équipe Mitochondries et Mort

Cellulaire, Cnrs UMR 5095-

Université de Bordeaux 2,

1, rue Camille Saint-Saëns,

33077 Bordeaux, France.

signal apoptotique. Bad, Bim et Bmf obéissent à une telle stratégie.

Dans une cellule non apoptotique, la protéine Bad est phosphorylée, via la voie impliquant la protéine kinase Akt/PKB et reste ainsi séquestrée par les protéines 14-3-3 cytosoliques. Lors d'une induction apoptotique, Bad n'est plus phosphorylée, son interaction avec 14-3-3 diminue et elle peut alors interagir avec $\mathrm{Bcl}-2$ ou $\mathrm{Bcl}-\mathrm{x}_{1}$ et ainsi inhiber leur action anti-apoptotique [2].

Les protéines Bmf et Bim sont quant à elles séquestrées au niveau du cytosquelette dans les cellules saines. Bmf est maintenue inactive par son interaction spécifique avec la chaîne légère 2 de la dynéine [3] et Bim par sa liaison avec la chaîne légère LC8 de la dynéine [4]. La perception d'un signal apoptotique détruit ces interactions et libère Bim et Bmf qui sont alors à même de neutraliser les protéines anti-apoptotiques. Il est intéressant de noter que le gène codant pour Bmf se trouve sur une région chromosomique qui est délétée dans de nombreux carcinomes métastatiques. L'absence de Bmf est donc directement corrélée à la capacité métastatique des cellules. Ceci suggère que l'identification d'un rôle propre de Bmf est en bonne voie : Bmf pourrait avoir un rôle physiologique dans les cellules saines, et son interaction avec le cytosquelette contribuerait au maintien de l'adhérence cellulaire.

La mieux connue des protéines de cette sous-famille de «protéines à $\mathrm{BH} 3$ seulement » reste Bid. Cette protéine de 22 kDa joue un rôle essentiel dans la 
mort des cellules dites de type II, dans lesquelles l'étape mitochondriale de l'apoptose est nécessaire à l'activation des caspases effectrices du programme. Dans ces cellules, l'induction apoptotique repose sur l'intervention des récepteurs de surface de la famille du TNFo, et provoque la formation limitée (restreinte?) du complexe DISC (death inducing signaling complex). Ce complexe recrute et active la pro-caspase 8 dont une des cibles est la protéine proapoptotique Bid. Le clivage de Bid in vivo semble être contrôlé par un phénomène de phosphorylation/déphosphorylation : Bid est un substrat des caséine kinases 1 et 2, et n'est pas reconnue par la caspase 8 lorsqu'elle est sous sa forme phosphorylée. Elle doit donc être déphosphorylée pour être clivée et activée par la caspase 8 [5]. La caspase 8 peut reconnaître plusieurs sites de clivage dans la protéine Bid mais produit majoritairement un fragment $\mathrm{C}$-terminal de 15 kDa (clivage après l'asp59) appelé tcBid (pour truncated c-terminal Bid). tcBid est capable d'interagir avec Bax pour favoriser les séquences d'événements aboutissant à l'activation de cette dernière : l'interaction avec tcBid facilite l'adressage de Bax aux membranes mitochondriales externes et provoque une modification dans la réactivité de Bax vis-à-vis d'anticorps conformationnels, suggérant une exposition de son domaine $\mathrm{N}$-terminal. $\mathrm{Ce}$ changement de conformation permet une réorganisation des monomères Bax sous forme d'oligomères et précède l'insertion de ces complexes dans la membrane externe de l'organite [6]. Bax est supposé acquérir ainsi son activité proapoptotique qui consiste à modifier la perméabilité de la membrane mitochondriale externe et se manifeste par la relocalisation de facteurs apoptogènes (cytochrome c, AIF, smac/DIABLO, endonucléase G) [7].

Plus récemment, il a été suggéré que tcBid ne soit pas qu'un simple activateur de Bax, mais qu'elle soit aussi compétente pour provoquer par ellemême la libération des protéines apoptogènes à partir de mitochondries iso-

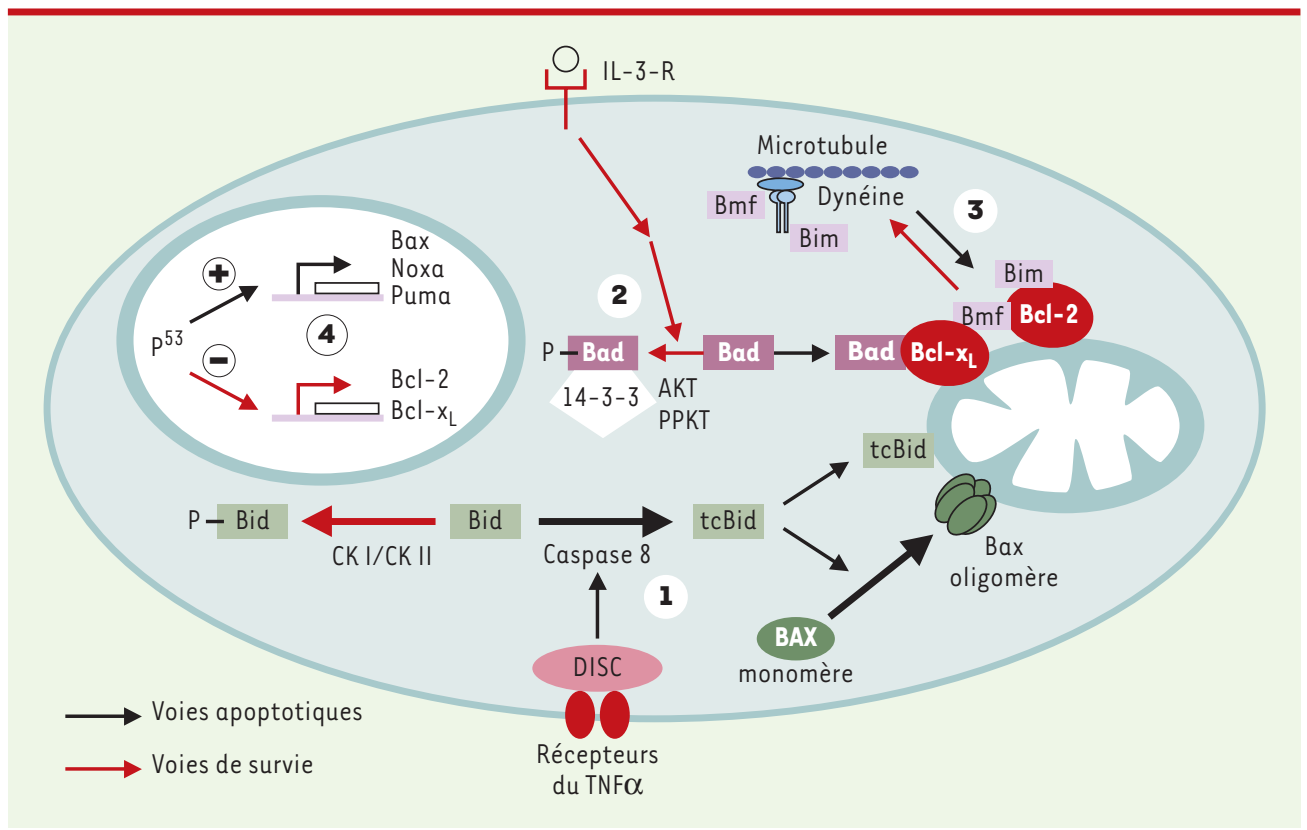

Figure 1. Les différents modes d'action des protéines à $\mathrm{BH} 3$ seulement. 1. L'activation de la famille des récepteurs du TNF induit une cascade de signalisation conduisant à l'activation de la protéine Bid en sa forme clivée tcBid. Cette protéine permet l'oligomérisation et l'insertion de Bax dans la membrane mitochondriale externe. La phosphorylation de Bid par les caséine-kinases I et II empêche son activation. 2. Dans les cellules non-apoptotiques, Bad est phosphorylée par la protéine-kinase Akt/PKB, sous contrôle des facteurs de survie de type interleukine3. Bad phosphorylée est maintenue dans le cytosol via son interaction avec les protéines 14-3-3. Le retrait des facteurs de survie empêche la phosphorylation de Bad, permettant ainsi son interaction inhibitrice sur les protéines anti-apoptotiques $\mathrm{Bcl}-2$ et $\mathrm{Bcl}-\mathrm{x}_{\mathrm{L}}$. 3. Dans les cellules non-apoptotiques, Bim et Bmf sont associés au cytosquelette par leur interaction avec la dynéine. La rupture de cette interaction, au cours de l'apoptose ou par déstabilisation du cytosquelette, permet à Bim et Bmf d'exercer une interaction inhibitrice avec les protéines anti-apoptotiques $\mathrm{Bcl}-2$ et $\mathrm{Bcl}-\mathrm{x}_{\mathrm{L}}$. 4. La protéine $\mathrm{p} 53$ règle négativement la transcription des gènes codant pour les protéines anti-apoptotiques $\mathrm{Bcl}-2$ et $\mathrm{Bcl}-\mathrm{x}_{\mathrm{L}}$, règle positivement la transcription du gène codant pour la protéine pro-apoptotique Bax mais aussi la transcription des gènes codant pour les protéines à BH3-seulement, telles que noxa et puma.
1. Gross A, Mc Donnell JM, Korsmeyer SJ. Bcl-2 family members and the mitochondria in apoptosis. Genes Dev 1999 ; $13: 1899-911$.

2. Zha J, Harada $H$, Yang $\varepsilon$, Jockel J, Korsmeyer SJ. Serine phosphorylation of death agonist Bad in response to survival factor results in binding to $14-3-3$ not $\mathrm{Bcl}-\mathrm{xl}$. Cell 1996; $87: 619-28$.

3. Puthalakath $H$, Villunger $A$, O'Reilly LA, et al. Bmf: a proapoptotic BH3-only protein regulated by interaction with the myosin $\mathrm{V}$ actin motor complex, activated by anoikis. Science 2001 . $293: 1829-32$.

4. Puthalakath $\mathrm{H}$, Huang DCS, O'Reilly LA, King SM, Strasser A. The proapoptotic activity of the Bcl-2 family member Bim is regulated by interaction with the dynein motor complex. Mol Cell $1999 ; 3: 287-96$.

5. Desagher $S$, Osen-Sand $A$, Montessuit $\mathrm{S}$, et al. Phosphorylation of Bid by casein kinases I and II regulates its cleavage by caspase 8 . Mol Cell 2000; 8: 601-11.

6. Eskes R, Desagher $S$, Antonsson B, Martinou JC. Bid induces the oligomerization and insertion of $B a x$ into the outer mitochondrial membrane. Mol Cell Biol 2000; 20: 929-35. 
7. Desagher S, Martinou JC. Mitochondria as the central control point of apoptosis. Trends Cell Biol 2000; 10: 369-77.

8. Kudla G, Montessuit $S$, Eskes $\mathrm{R}$, Berrier C, Martinou JC, Ghazi A, Antonsson B. The destabilization of lipid membranes induced by the $C$-terminal fragment of caspase 8 -cleaved bid is inhibited by the $\mathrm{N}$-terminal fragment J Biol Chem 2000; 275: 22713-8.

9. Basañez G, Nechushtan A, Drozhinin 0, et al. Bax, but not $B c l-x_{L}$, decreases the lifetime of planar phospholipid bilayer membranes at subnanomolar concentrations. Proc Natl Acad Sci USA 1999; 96: 5492-7.

10. Fesik SW. Insights into programmed cell death through structural biology. Cell 2000; 103: 273-82.

11. Matsuyama S, Nouraini S, Reed JC. Yeast as a tool for apoptosis research. Curr Opin Microbiol 1999; 2: 618-23. lées [8]. L'ensemble de ces résultats a donc suggéré que la fonction pro-apoptotique de tcBid in vivo repose sur ses capacités conjointes d'interagir avec Bax par un mécanisme dépendant de $\mathrm{BH} 3$, et de déstabiliser les membranes lipidiques par un mécanisme semblable à celui décrit pour Bax [9].

La fonction des autres protéines «à $\mathrm{BH}-3$ seulement » reste mal connue. Pour certaines d'entre elles (noxa, puma), le fait qu'elles soient réglées positivement par le facteur de transcription p53 suggère cependant un rôle important dans le déroulement du programme apoptotique.

A mesure que la recherche avance, le programme génétique responsable de la mort cellulaire par apoptose apparaît donc d'une admirable sophistication. II recrute des partenaires organisés en une famille de protéines qui interviennent dans la plupart des compartiments cellulaires, et dont les fonctions s'enchevêtrent en un réseau apparemment inextricable. Une telle complexité est probablement le garant de la capacité cellulaire de s'engager dans l'apoptose par des voies multiples, mais aussi de ne pas déclencher le programme quand il s'agit de répondre à des stress mineurs.

De quels outils disposons-nous alors pour pallier les dysfonctionnements d'un si vaste et si complexe écheveau ? L'élucidation du fonctionnement individuel de chacune des composantes apparaît comme un pré-requis indispensable. Les outils permettant l'étude de la fonction individuelle de ces protéines, tels que la biochimie structurale [10], le développement de modèles d'apoptose in vitro [7] ou l'utilisation de «tubes à essai cellulaires » comme la levure Saccharomyces cerevisiae [11], deviennent indispensables pour que la mise en place des modèles de régulation s'accompagne d'une compréhension de ces phénomènes au niveau moléculaire, capable d'apporter les informations requises pour de nouvelles avancées thérapeutiques. $\Delta$

BH3 only proteins of the Bcl-2 family

\section{NOUVELLE}

AIF, le facteur inducteur
de "apoptose, est tenu
en échec par la protéine
de stress Hsp70
Luigi Ravagnan, Sandeep Gurbuxani, Carmen Garrido,
Guido Kroemer

$>$ Les protéines de choc thermique (heat shock proteins, HSP), définies à l'origine pour leur rôle cytoprotecteur contre le choc thermique, constituent une classe de protéines très conservées dans l'évolution. Par la suite, ces protéines se sont avérées protéger les cellules contre un grand nombre d'autres agressions chimiques aussi bien que physiques, et ce par leur inhibition de l'apoptose $[1,2]$. Toutefois, les cibles moléculaires des HSP, en particulier celles de Hsp70 intervenant dans les voies d'induction de l'apoptose, ne sont connues que très partiellement. Deux équipes ont démontré de façon indépendante que Hsp70 peut interférer avec la formation du complexe activateur des caspases dénommé «apoptosome», composé du cytochrome $c$, de la protéine adaptatrice Apaf-1 (apoptotic protein activating factor-1) et de I'ATP. Hsp70 séquestre Apaf-l et l'em-
L. Ravagnan, G. Kroemer : Cnrs, UMR 1599,

Institut Gustave Roussy, Pavillon de Recherche 1, 39 , rue Camille Desmoulins, 94805 Villejuif, France.

S. Gurbuxani, C. Garrido : Inserm U517, Faculté de Médecine et de Pharmacie, 7, boulevard Jeanne d'Arc, 21033, Dijon, France. pêche de recruter la procaspase 9 au niveau de l'apoptosome, bloquant ainsi la cascade apoptotique dépendante des caspases [3, 4]. Apaf-1 n'est toutefois pas toujours nécessaire à l'effet cytoprotecteur de Hsp70 : nous avons montré en effet que la surexpression stable de Hsp70 dans des cellules

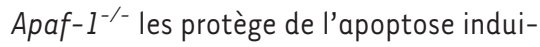
te par la carence en facteurs de croissance [5]. Or, AIF (apoptosis inducing factor), un effecteur de la mort qui ne met pas en jeu la voie des caspases [6] s'est révélé indispensable, entre autres, dans le processus d'apoptose induit par la caren- 